\title{
Scale invariance of morphogen-mediated patterning by flux optimization
}

\author{
David M. Umulis \\ Department of Ag. and Biological Engineering \\ Weldon School of Biomedical Engineering \\ Purdue University \\ West Lafayette, IN 47907 USA \\ email: dumulis@purdue.edu
}

\author{
Hans G. Othmer \\ School of Mathematics \\ University of Minnesota \\ Minneapolis, MN 55455 USA \\ email: othmer@math.umn.edu
}

\begin{abstract}
The development of an organism from a single cell into an adult requires exquisite control of spatial patterning so that the correct tissues are formed at the correct place and at the correct time. A number of molecular factors that regulate spatial patterning of tissues during development have been identified, however, relatively little is known about how they form consistent spatial patterns of cell signaling or gene expression that scales in proportion to the size of the tissue being patterned. In the model organism Drosophila melanogaster, embryonic morphogen gradients establish the anterior/posterior (AP) and dorsal/ventral (DV) axes to establish the boundaries of gene expression that are further subdivided into distinct cell types. Both AP and DV patterning systems exhibit a high degree of scaling, however, they have evolved distinct mechanisms to achieve scaling. Our analysis herein shows that recently discovered data for Bicoid (the principle AP morphogen) embryonic patterning is consistent for a mechanism that optimizes the flux of Bcd molecules into the anterior end. This mechanism is distinct from mechanisms suggested in other systems that target biophysical properties that regulate morphogen range, thereby stretching or shrinking the morphogen distribution accordingly. Instead, flux optimization changes the morphogen peak levels to provide "approximate" scaling over a large region of space and perfect scaling at a single spatial location. While remarkably simple, this mechanism of scale-invariance is sufficient for increases and decreases in tissue size by $\mathbf{5 0 \%}$ and confers less than $10 \%$ error in spatial patterning.
\end{abstract}

\section{INTRODUCTION}

As more information becomes available about the various molecules required for the orderly patterning and signal transduction during development, there is an increasing need to understand how such components are integrated into networks and how the networks process the molecular information to regulate gene expression and the phenotype of an organism during development. An important question that has recently seen a surge in experimental and mathematical analysis is: How do patterns of gene expression scale in proportion to the tissue to preserve proportions? This question, appears to have many solutions and herein we present a new mechanism that can account for proper scaling in a number of developing systems.

The principle paradigm for understanding the processes that give rise to spatial patterns during development was proposed over half a century ago by Alan Turing in a field defining paper that discussed how spatial patterns can be generated by reaction and diffusion of chemical compounds he termed morphogens [1]. Morphogens are secreted molecules that form a spatially non-uniform distributions and cells interpret the morphogen information by either modifying gene expression or by altering the cell's intracellular state (e.g. initiating a signaling cascade).

Morphogens form spatial patterns in tissues by a balance of dynamic processes that regulate molecular transport by advection or diffusion, chemical reactions such as binding and decay, and the rate of morphogen secretion by a source. The physical processes of transport and reaction contain an inherent time-scale and the ratio of time-scales between reaction and transport dictate the morphogen range. Often, the transport and reaction rates are intensive variables and therefore do not depend on the size or length of the system. This is problematic because the range of the morphogen would be governed only by the physical processes that are constant irrespective of changes in tissue or organism size. Left alone, a morphogen patterning system would not adjust to accommodate for differences in size between individuals or in a growing tissue.

Instead, mechanisms have evolved in biology so that the patterning process is affected by the size of the tissue or organism being patterned and this can lead to scale-invariance (Figure 1 A,B). Recently, we have shown how modification of binding site density for secreted diffusible morphogens can automatically adjust the effective diffusion and decay rates to ensure scaling of the morphogen spatial distributions. This mechanism is remarkably simple, yet effective, at scaling morphogen gradients but new biology has emerged to suggest an alternative scaling mechanism that is "good enough" over a relatively wide range of tissue and embryo sizes in the model organism Drosophila melanogaster.

Drosophila early development proceeds through sequential stages of nuclear division in a syncytial embryo (a multi-nuclei single cell). Dynamic patterning prior to fertilization place maternal RNA transcripts for the anterior/posterior determinant Bicoid (Bcd) near the anterior pole of the embryo. Other maternal RNAs are placed that also participate in embryonic patterning, primarily through their integrated control of down- 
A

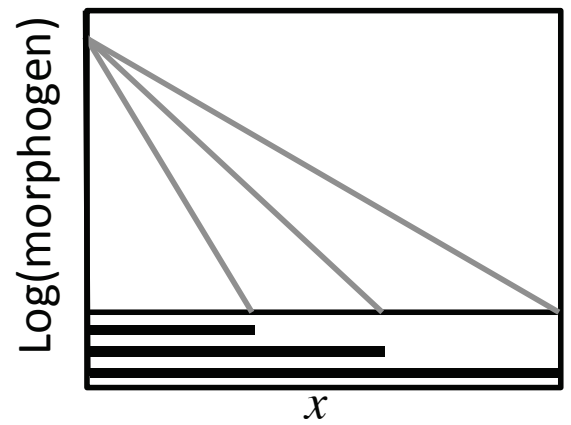

B

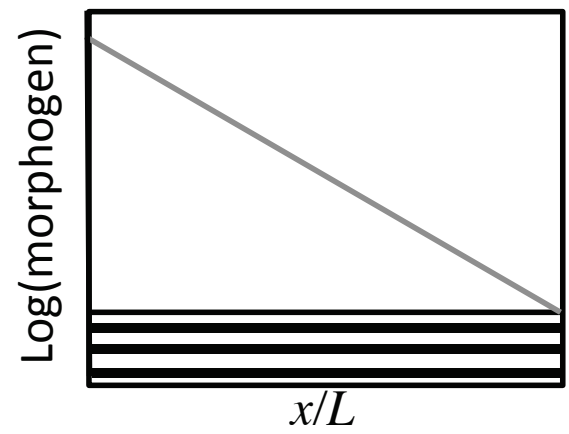

C

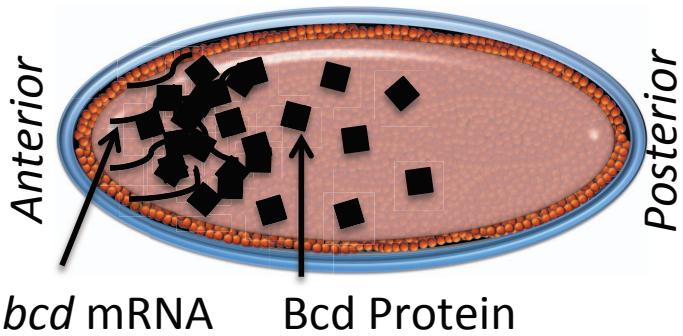

Fig. 1. Scale invariance of Bcd-mediated patterning. (A-B) Example morphogen profiles for a system that exhibits scale-invariance. (A) Morphogen profile for scaling as a function of absolute position in three hypothetical tissues with relative lengths shown by black bars (bottom). (B) Same as in (A), except replotted with normalized position $x / L$. (C) Schematic of an early Drosophila embryo for illustrative purposes shows approximate position of mRNA and Bcd protein.

stream genes called the gap genes. Shortly after fertilization, translation of the maternal $b c d$ mRNAs produces Bcd protein, a transcription factor that is transported throughout the embryo by diffusion and potentially other transport processes (Figure 1C).

The Bcd target, hunchback mRNA is both maternallyinherited and transcribed in the zygote in response to the local level of Bcd protein and other gene products. High Bicoid levels in the anterior activate zygotic transcription of hunchback. In the next stage, which is initiated by Bicoid and Hunchback, the AP axis is divided into several broad regions by the expression of the gap genes giant, kruppel, and knirps. Thus, the initial patterns established by Bcd presage downstream gap gene expression boundaries and ultimately the anterior-posterior patterning of tissues needed for normal development.

The initial Bcd protein distribution is "exponential-like" consistent with a patterning mechanism whereby Bcd is secreted in a localized region, moves by diffusion, and undergoes linear decay or capture [2], [3]. At the same time, nuclei in the embryo undergo 13 cycles of synchronous division, and during cycle 10 translocate from the core to the cortex, where they remain for the next rounds of nuclear division. During cycle 14 there are about 6,000 nuclei localized at the embryonic cortex, and at this stage membranes form and segregate the syncytial nuclei into individual cells [4]. The simplest model for Bcd patterning during the syncytial blastoderm stage is termed the synthesis-diffusion-decay (SDD) model. The SDD Bcd model has been the most widely used, although not yet generally accepted, mechanism for Bcd transport and gradient formation in the embryo.

Experimental evidence supports two types of scaling mechanisms for Bcd-mediated embryonic patterning. Primary evidence for Bcd scaling first appeared in 2005 in a study that investigated Bcd gradient formation in closely related species of Diptera [3]. It was shown by immunostaining that the distribution of Bcd stretches or contracts in proportion to the average embryo size for each species. Specifically, the Bcd distributions in species that ranged in size from $\sim 344$ microns (D. busckii) to $\sim 1420$ microns (L. sericata), appear to expand in direct proportion to the length of the embryo (cf Figure 1). When rescaled to normalized coordinates - either percent embryo length, or equivalently $\xi=x / L-$ the interspecies variation of the profiles is no larger than the intraspecies variations, and thus the relative positions of target gene expression patterns would be unchanged and on a graph of Bcd concentration vs. normalized position, the profiles for Bcd protein are superimposed and nearly indistinguishable [3].

Intraspecies scaling of Bcd patterns appear to rely on a completely different mechanism than between species scaling. Recently Cheung et al., [5] investigated the spatial patterns of Bcd patterning between artificially selected populations of an initially wild type Drosophila melanogaster. After successive rounds of selection, two populations were developed that differed in egg length by about $25 \%$. Small embryos with a length of $\sim 518$ microns and large embryos with a length of $\sim 645$ microns yielded Bcd patterns that had the same intrinsic length scale for the Bcd variation. Since the intrinsic scale is not changed, perfect invariance is not possible, but approximate scaling could result from modulation of the amplitude. A hint of an alternative mechanism emerged from careful quantification of the Bcd distributions in populations of large and small embryos that showed that the amplitude (the maximum value) of the Bcd distribution increased by approximately $66.9 \%$, a number that correlated with an embryo volume difference of $71.7 \%$.

\section{RESULTS}

\section{A. The governing equations}

The distribution of Bcd by the SDD mechanism can be described by a linear PDE with constant coefficients. Suppose 
that the spatial domain is the interval $[0, L]$, that transport is via diffusion only, and that there is an input flux at $x=0$ and a no flux boundary at the distal postion $x=L$. The distribution of the Bicoid morphogen (denoted $m$ for morphogen) can be described as follows:

$$
\frac{\partial m}{\partial t}=\frac{\partial}{\partial x}\left(D_{m} \frac{\partial m(x, t)}{\partial x}\right)-k_{m} m .
$$

with the boundary and initial conditions

$$
\begin{aligned}
-D_{m} \frac{\partial m(x, t)}{\partial x} & =j, \quad x=0 \\
\frac{\partial m(x, t)}{\partial x} & =0, \quad x=L \\
m(x, 0) & =m_{0}(x), \quad x \in(0, L),
\end{aligned}
$$

where $D_{m}$ is the diffusion coefficient for $\mathrm{Bcd}, k_{m}$ is the linear decay rate for $\mathrm{Bcd}$, and $j$ is the input flux of Bcd.

Scaling the position by the system length $\xi=x / L$, setting $\lambda^{2}=\left(k_{m} L^{2}\right) / D_{m}$ and $J=(j L) / D_{m}$ and assuming that diffusion and decay are time-invariant and spatially uniform leads to the dimensionless form of the equations:

$$
\begin{aligned}
\frac{\partial^{2} m}{\partial \xi^{2}} & =\lambda^{2} m \\
-\frac{\partial m}{\partial \xi}(0) & =J \\
\frac{\partial m}{\partial \xi}(1) & =0
\end{aligned}
$$

This has the solution:

$$
m(\xi)=\frac{J}{\lambda}\left[\frac{\exp (\lambda(2-\xi))+\exp (\lambda \xi)}{\exp (2 \lambda)-1}\right]
$$

and for large $\lambda$ (large $k_{m}$, small $D_{m}$ ), this can be simplified to:

$$
m(\xi) \approx \frac{j}{\sqrt{k_{m} D_{m}}} \exp \left(-\sqrt{\frac{k_{m} L^{2}}{D_{m}}} \xi\right) .
$$

By inspection of equation 10, to achieve scaling, the ratio of terms in the exponential need to lose their L-dependence. In effect, scale-invariance of the spatial pattern can be achieved by speeding up diffusion, slowing the reaction or a combination of these to eliminate $L^{2}$. However, appropriate changes must also be made to the input flux $j$, lest the profile will increase or decrease in amplitude depending on whether diffusion or decay rates are modified. We have previously analyzed this system in detail and refer the interested reader to Othmer et al., 1980, Umulis et al. 2008 and Umulis 2009 [6], [7], [8]. Herein we focus on an alternative and remarkably simple mechanism that confers approximate scale invariance in Drosophila embryos.
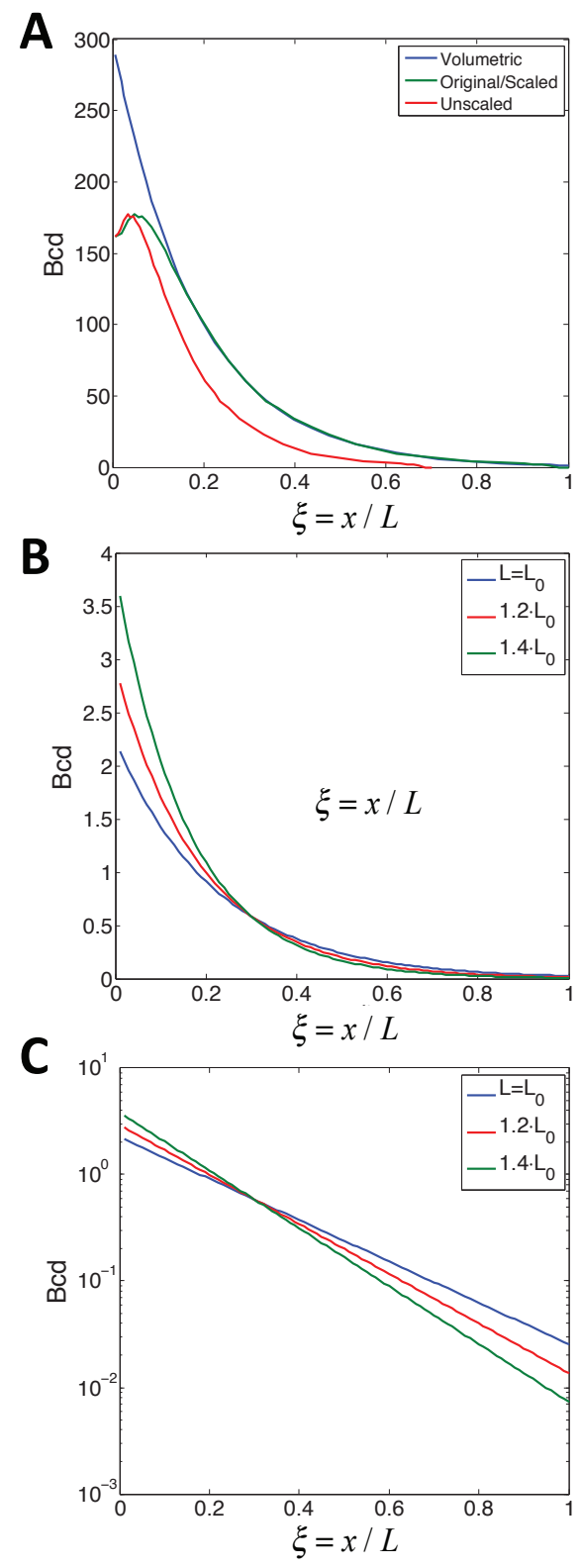

Fig. 2. Scaling of Bcd by adjustment of morphogen range or by a volume-dependent production rate of Bcd. (A) Relative Bcd levels (small embryos represent the base case, which is the green line) along the AP axis for the volume-dependent production mechanism (blue), a hypothetical perfect scaling mechanism (green), and for no scaling of Bcd (red). (B-C) Predicted Bcd distributions under the flux optimization hypothesis targeted at a relative spatial position of 0.3 on a linear (B) or $\log (\mathrm{C})$ scale.

\section{B. Scaling by flux optimization}

Increasing or decreasing the amplitude may provide enough scaling to meet the performance objectives of development in systems that do not modulate the intrinsic scale, and this may suffice for relatively small increases and decreases in length. If patterning occurs by interpretation at only one threshold concentration, an increase or decrease in the source strength $j$ can lead to the same concentration at the same relative 

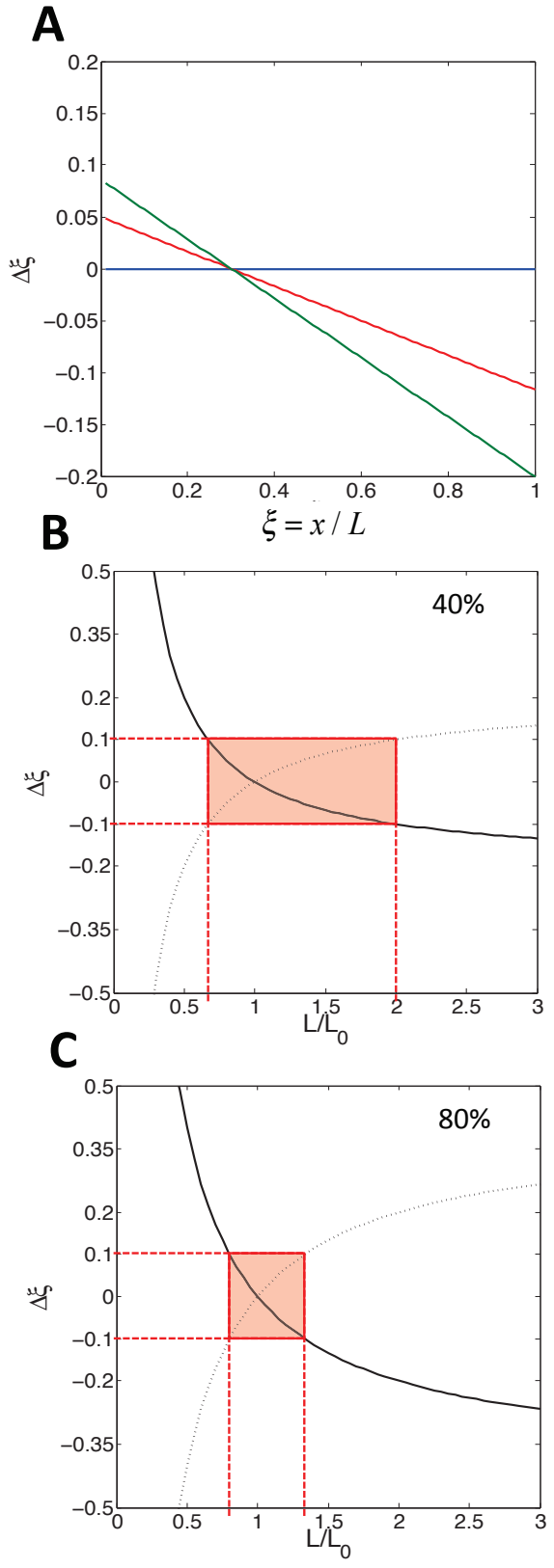

Fig. 3. Analysis of flux optimization mechanism. (A) Plot of positional error as a function of relative spatial position along the embryo axis. (B-C) Range of effectiveness of flux-optimization mechanism that allows for $+/-10 \%$ error in spatial position near the threshold. (E) Length range for flux optimal scaling at the threshold position and positions $+/-20 \%$ of the total length. (C) Same as (B) except for scaling at the threshold position and position at $+/-$ $40 \%$ of the total length.

spatial location. Thus if the concentration of Bcd at only once spatial position was needed to trigger a boundary, for instance, then perfect scaling could always be achieved by adjusting the input flux to raise or lower the overall profile by the amount needed by the target. Flux or concentration optimization simply supposes that the input secretion rate of morphogen increases in larger tissues and decreases in smaller tissues to a level optimized for the specific patterning process. We speculate that the relatively small changes in flux needed for flux optimization already exist in most populations of Drosophila, allowing for the short term and rapid evolution of artificially selected populations of large embryos and small embryos [9].

To analyze scaling by flux optimization, suppose that there is a critical threshold in the gradient (e.g. the boundary of Hbk gene expression) that occurs at $\xi_{T}$. Then the flux increase required for scaling at $\xi_{T}$ can be calculated by equation 11:

$$
j_{L}(L)=j_{0} \exp \left(\sqrt{\frac{k_{m}}{D_{m}}}\left(L-L_{0}\right) \xi_{T}\right) .
$$

Taking the data from Cheung et al. with $\left(D_{m} / k_{m}\right)^{1 / 2}=99$ microns [5], assuming $\xi_{T}=0.4$ is the spatial location where the critical threshold is crossed in the embryo (near the Hunchback boundary), and using $L=645$ for the large embryo and $L_{0}=518$ for the small embryo, the calculated optimal flux $j_{L}$ is $67 \%$ greater in the large embryo than the flux in the small embryo $j_{0}$. This is remarkably close to the increase in amplitude measured for Bcd scaling of $66.9 \%$. Thus, while the amplitude correlates with embryo volume, it also strongly correlates with a flux or concentration optimization process. The error at other spatial positions away from the critical threshold can be calculated by:

$$
\Delta \xi=\xi_{L}-\xi=\left(1-\frac{L_{0}}{L}\right)\left(\xi_{T}-\xi\right) .
$$

Depending on the allowable variations in the spatial positions of gene expression $\Delta \xi$, equation 12 can be used to estimate the range of lengths where flux optimization provides sufficient scaling.

We found that increasing the amplitude of Bcd in accord with observations by Cheung et al., yields the profiles for Bcd protein shown in Figure 2A. An increase of 66.9\%, keeping the decay length fixed, yields a gradient that is nearly indistinguishable from the wt distribution for approximately $80 \%$ of the length. Gap gene interpretation, an example downstream feedback system, yields patterns of target genes that are equivalent to the shorter embryos with only a few minor defects in patterning, as observed experimentally [10]. The amplitude increase performs as well as the perfect case in which the Bicoid profile scales uniformly (row 3). The unscaled Bcd input yields incorrect gap gene placement and ectopic banding, among other defects (our unpublished data).

To estimate the range of embryo sizes over which amplitude scaling is sufficient, the input flux was optimized so that it could scale precisely at one spatial position and imprecisely at all other spatial positions. If the spatial position of $\xi=0.3$ is selected, flux optimization produces profiles that cross at $\xi=0.3$ and differ from each other at all other spatial positions (Figure 2B,C). The error in positional information at all other spatial positions $\xi$ can be calculated giving the error estimate in spatial patterning $\Delta \xi$ shown in Figure 3A. As can be seen there, for $\xi=0.3$ there is less than a $10 \%$ error in 
spatial position for thresholds between $\xi=0$ and $\xi=0.9$ for a $20 \%$ increase in embryo length (red line) or between $\xi=0$ and $\xi=0.6$ for a $40 \%$ increase in length (green line). If a positional error of $10 \%$ is chosen to be the maximal allowable error, increasing the production of morphogen (thus increasing the amplitude) can lead to scale-invariance for a range of lengths. Figure $3 \mathrm{~B}-\mathrm{C}$ show that positional error is less than $10 \%$ for lengths that range from $60 \%$ to $200 \%$ of the original length (when $40 \%$ of total morphogen spatial range is used) or from approximately $70 \%$ to $140 \%$ of the original length (when $80 \%$ of the morphogen spatial range is used). Thus amplitude scaling provides a simple mechanism to correct for small increases and decreases in length that might occur between individuals within a species, whereas between species, the measured range of Bcd suggests that the properties of the morphogen patterning system are tuned to confer scaling. In flux optimization systems, precise scaling of one threshold position results in either hyper- or hypo- scaling at all other spatial positions, but the relative error might be small enough that the system as a whole can tolerate imprecise scaling (Figure 2A-C; Figure 3A-C) [11].

\section{CONCLUSiON}

The biological solutions to scaling of developmental patterns have evolved in diverse ways that target physical processes. Much interest has been placed on understanding how biochemical mechanisms evolved that allow for the adjustment of transport and reaction rates to adjust the final distributions of morphogens [3], [12], [13], [8]. This seems to be the preferred method for large changes in embryo or tissue size that occurs during growth or in equivalent tissues between different species. For relatively small changes of less than $50 \%$ in total length, sufficient scaling can be achieved by simply adjusting the amount of morphogen supplied to the system. While the shapes of the distributions differ, there remains a large spatial domain where the error in position is less than $10 \%$ for exponential-like gradients. It will be interesting to see how multiple mechanisms are integrated to tolerate both large changes in tissue or organism size and smaller changes in size that exist between individuals in a population. While the flux optimization mechanism is consistent with published experimental results, the mechanism that adjusts the flux for different size embryos remains unknown. In Bicoid mediated patterning the issue is even more intriguing since the source of Bicoid protein originates from maternally deposited mRNA.

\section{ACKNOWLEDGMENT}

The authors would like to thank National Institutes of Health grant R01GM029123-28 to HGO and the University of Minnesota subaward 529626301 to DMU.

\section{REFERENCES}

[1] A. M. Turing, "The chemical basis of morphogenesis," Philosophical Transactions of the Royal Society of London. Series B, Biological Sciences, vol. 237, no. 641, pp. 37-72, 1952.

[2] B. Houchmandzadeh, E. Wieschaus, and S. Leibler, "Establishment of developmental precision and proportions in the early Drosophila embryo," Nature, vol. 415, no. 6873, pp. 798-802, 2002.

[3] T. Gregor, W. Bialek, R. R. de Ruyter van Steveninck, D. W. Tank, and E. F. Wieschaus, "Diffusion and scaling during early embryonic pattern formation," Proc Natl Acad Sci U S A, vol. 102, no. 51, pp. 18403-7, Dec. 202005.

[4] V. E. Foe, B. M. Alberts et al., "Studies of nuclear and cytoplasmic behaviour during the five mitotic cycles that precede gastrulation in drosophila embryogenesis," J. Cell Sci, vol. 61, no. 3, pp. 1-70, 1983.

[5] D. Cheung, C. Miles, M. Kreitman, and J. Ma, "Scaling of the bicoid morphogen gradient by a volume-dependent production rate," Development, vol. 138, no. 13, pp. 2741-2749, 2011.

[6] H. G. Othmer and E. F. Pate, "Scale invariance in reaction-diffusion models of spatial pattern formation," Proc. Nat. Acad. Sciences, vol. 77, pp. 4180-4184, 1980.

[7] D. Umulis, M. B. O'Connor, and H. G. Othmer, "Robustness of embryonic spatial patterning in Drosophila melanogaster," Current Topics in Developmental Biology, vol. 81, pp. 65-111, 2008.

[8] D. M. Umulis, "Analysis of dynamic morphogen scale invariance," Journal of The Royal Society Interface, vol. 6, no. 41, pp. 1179-1191, 2009.

[9] C. M. Miles, S. E. Lott, C. L. L. Hendriks, M. Z. Ludwig, C. L. Williams, M. Kreitman et al., "Artificial selection on egg size perturbs early pattern formation in drosophila melanogaster," Evolution, vol. 65, no. 1, pp. 3342, 2011.

[10] A. M. De Lachapelle and S. Bergmann, "Precision and scaling in morphogen gradient read-out," Molecular Systems Biology, vol. 6, no. 1, 2010.

[11] S. E. Lott, M. Kreitman, A. Palsson, E. Alekseeva, and M. Z. Ludwig, "Canalization of segmentation and its evolution in drosophila," Proceedings of the National Academy of Sciences, vol. 104, no. 26, p. 10926, 2007.

[12] O. Wartlick, P. Mumcu, A. Kicheva, T. Bittig, C. Seum, F. Julicher, and M. González-Gaitán, "Dynamics of dpp signaling and proliferation control," Science, vol. 331, no. 6021, pp. 1154-1159, 2011.

[13] D. Ben-Zvi, G. Pyrowolakis, N. Barkai, and B. Z. Shilo, "Expansionrepression mechanism for scaling the dpp activation gradient in drosophila wing imaginal discs," Current Biology, vol. 21, pp. 13911396, 2011. 